\title{
Two-Year Follow-Up After Percutaneous Dilatational Tracheostomy in a Surgical ICU
}

\author{
Maria Theresa Voelker MD, Marcus Wiechmann MD, Andreas Dietz MD, Sven Laudi MD MA, \\ and Sven Bercker PhD
}

\begin{abstract}
BACKGROUND: The modalities of tracheostomy for critically ill patients are still controversially discussed. Although the use of percutaneous dilatational tracheostomy (PDT) is generally accepted to be a safe procedure, it is still not considered to be suitable for patients requiring a permanent tracheostomy. METHODS: This was an observational cohort study investigating long-term outcome parameters of PDT. All patients having received a PDT at the interdisciplinary ICU at the University of Leipzig between October 2008 and August 2009 that survived to discharge were asked for consent to participate $(n=103)$. Baseline data, admitting diagnosis, reasons for tracheostomy, and timing for tracheostomy were recorded. Subjects and medical providers were asked for neurological outcome, adverse events, and personal discomfort related to the tracheostomy. The study period was $2 \mathrm{y}$ from the time of tracheostomy. RESULTS: Seventy-one subjects were finally included in the survey. Data of 8 subjects $(11 \%)$ were incomplete. Reasons for tracheostomy were the need for long-term ventilation $(42 \%)$, weaning $(31 \%)$, and neurological deficits $(27 \%)$. No severe adverse events were registered. Sixty-five percent of all subjects could finally be decannulated. Two subjects remained with their initial tracheostomy, and in 5 subjects $(7 \%)$ tracheostomy was modified to a surgical tracheostomy. Thirty-seven percent of subjects died during the $2 \mathbf{y}$. Twenty-five of the surviving subjects (35\%) had only minor or moderate neurological and psychological deficits. Complaints were mostly connected to swallowing and breathing. CONCLUSIONS: Decannulations after PDT are easily done. Complications after PDT requiring a transformation to a surgical tracheostomy are rare. Elective surgical conversions are not necessary. Key words: tracheostomy; follow-up study; quality of life; complications. [Respir Care 2017;62(7):963-969. ( 2017 Daedalus Enterprises]
\end{abstract}

\section{Introduction}

Tracheostomy is a common procedure in ICUs. Patients with severe head trauma or intracranial bleeding often re-

Drs Voelker, Wiechmann, Laudi, and Bercker are affiliated with the Department of Anesthesiology and Intensive Care Medicine, and Dr Dietz is affiliated with the Clinic of Otolaryngology, Head and Neck Surgery, University of Leipzig, Leipzig, Germany.

The authors have disclosed no conflicts of interest.

Drs Voelker and Wiechmann contributed equally to this work.

Correspondence: Maria Theresa Voelker MD, Department of Anesthesiology and Intensive Care Medicine, University of Leipzig, Liebigstrasse 20, D-04103 Leipzig, Germany. E-mail: theresa.voelker@medizin.uni-leipzig.de.

DOI: $10.4187 /$ respcare. 05290 quire tracheostomy due to long-term ventilatory support or neurological deficits. ${ }^{1,2}$ It secures the airway while decreasing airway resistance and allowing for ventilator-free intervals. It also facilitates airway suction, enhances patients' mobility and comfort through reduced sedation, and gives an opportunity for verbal communication and oral food intake. ${ }^{3}$ Common approaches for tracheostomy can be divided into percutaneous and surgical techniques. However, apart from indication and the correct timing for tracheostomy in the critically ill, the superiority of one or another technique is still controversially discussed. ${ }^{4,5}$ Surgical tracheostomy is one of the oldest surgical procedures, first described in the first century $\mathrm{BC}$, and it is considered to be the safest way to perform a tracheostomy. However, a variety of studies and metaanalyses could not show distinct benefits for this practice. On the contrary, it seems that there are signifi- 
cantly fewer major bleeding events and stoma infections associated with percutaneous dilatational tracheostomy (PDT). ${ }^{6}$ Further, PDT can be performed much faster and has an easy bedside application, eliminating the risks associated with transport to the operating theater. Even risks classically associated with percutaneous procedures, such as tracheal stenosis, false routes, and pneumothoraxes, were not found to be higher in patients who underwent PDT. Nonetheless, uncertainty remains, and about one quarter of all tracheostomies are still performed surgically. ${ }^{7}$ One generally acknowledged reason to choose the surgical procedure over the percutaneous one is the possible need for a permanent tracheostomy. However, data on long-term outcomes of patients who have received a PDT are scarce.

\section{Methods}

This was a non-interventional observational cohort study including all patients who had received a PDT at the interdisciplinary surgical ICU of the University of Leipzig between October 2008 and August 2009 who were discharged alive. The study protocol was approved by the ethics committee of the Medical Faculty of the University of Leipzig and was conducted in accordance with the Ethical Principles of the Declaration of Helsinki. Individual consent was acquired from every subject or legal custodian before inclusion into the study. Baseline data concerning age, body mass index, severity of disease scores, and information on the tracheostomy and stay were gathered from the hospital's information system (SAP [SAP SE, Walldorf, Germany] and COPRA [COPRA System $\mathrm{GmbH}$, Berlin, Germany]). For severity of disease, we calculated APACHE II (Acute Physiology And Chronic Health Evaluation II) and SOFA (Sequential Organ Failure Assessment) scores and the SAPS II (Simplified Acute Physiology Score II). Subjects or legal custodians were asked to complete a questionnaire about their current situation, state of recovery, complaints concerning the tracheostomy or post-decannulation status, and feeding modalities. All answers from the questionnaires were analyzed anonymously. We further asked the post-acute health-care provider for further information on medical issues and adverse events connected to the tracheostomy. The overall neurological outcome was measured by the Glasgow outcome scale (Table 1 ). ${ }^{8}$ All data were collected in an Excel database (Excel 2010, Microsoft Corp, Redmond, Washington) using a random number system for anonymization. Results are expressed as mean \pm SD for normally distributed data and as median and interquartile range for ordinal data.

\section{QUICK LOOK}

\section{Current knowledge}

Bedside techniques for percutaneous dilatational tracheostomy (PDT) belong to the most common surgical procedures in the intensive care setting are generally acknowledged to be safe procedures. PDTs are not considered to be suitable for patients requiring a permanent tracheostomy. Especially in intensive care patients, care providers cannot know whether a tracheostomy will be permanent or provisional. Personal comfort, technical and medical difficulties during the following home-care are considered to be an important factor for the choice of technique for permanent tracheostomies.

\section{What this paper contributes to our knowledge}

In our 2 year follow-up of subjects who had received a PDT in the intensive care setting, most subjects could successfully be decannulated within the first 6 months. Subjects had a high frequency of common but not severely impairing symptoms after tracheostomy. No severe complications were reported after discharge of subjects with PDT during the following 2 years. Very few subjects underwent subsequent surgical tracheostomy. PDT is suitable for patients in the intensive care setting, even if the patient may eventually require a long-term tracheostomy.

\section{Results}

\section{Study Inclusion and Baseline Data}

Between October 2008 and August 2009, we performed 151 PDTs. Forty-eight tracheotomized patients (32\%) died during their hospital stay. Of the remaining 103 eligible patients $9(6 \%)$ were lost to follow-up after discharge. All remaining patients were asked for consent to participate in the study. Twenty-three (15\%) patients denied consent, and we were finally able to include 71 subjects in the survey, which equates to a return rate of $47 \%$. After inclusion, baseline information could be obtained from all 71 subjects. Information on the tracheostomy, survival, and living circumstances from the medical provider or legal custodian could also be acquired for all 71 subjects. However, the data on these information of 8 subjects were incomplete. Personal complaints obtained by either the return of a written questionnaire or telephone interview were completed by 61 subjects (Fig. 1).

Subjects' baseline data can be found in Table 2. Differences between survivors and non-survivors were found for age, APACHE II score, and SAPS II $(P<.001)$. Overall 
Table 1. Glasgow Outcome Scale for the Assessment of Persisting Disability After Brain Damage

\begin{tabular}{ll}
\hline \hline GOS & \multicolumn{1}{c}{ Functional Status } \\
\hline 1 & Death \\
2 & Persistent vegetative state \\
3 & Severe disability \\
4 & Moderate disability \\
5 & Good recovery \\
\hline GOS = Glasgow Outcome Scale. ${ }^{8}$ & \\
\hline
\end{tabular}

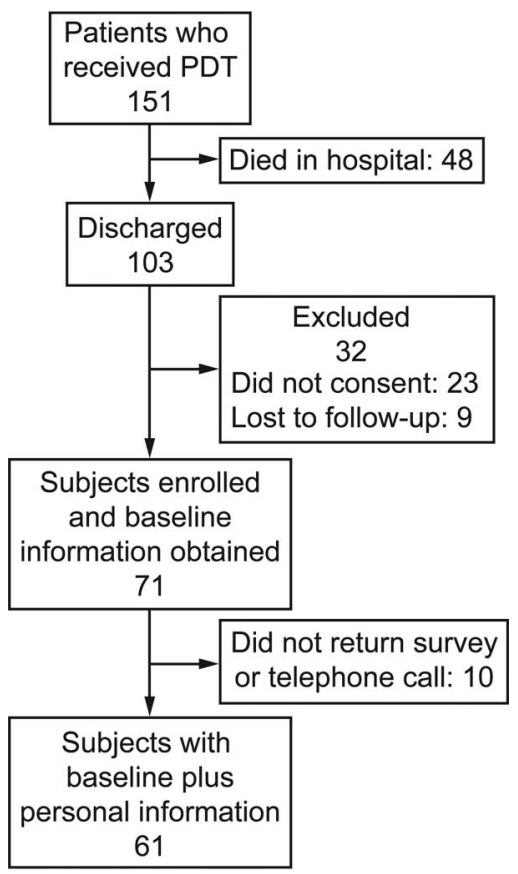

Fig. 1. Flow chart. PDT = percutaneous dilational tracheostomy.

2-y survival of all 151 tracheotomized subjects was $49 \%$ $(n=74)$. Survival of all subjects with completed follow-up after discharge was $63.4 \%$ (45 of 71).

\section{Tracheostomy}

Reasons for tracheostomy may be found in Table 2 . Thirty-eight subjects (53\%) were tracheotomized during their first $8 \mathrm{~d}$ on the ventilator and all others afterward. All tracheostomies were performed with a bedside technique using a single-dilator technique (Ciaglia Blue Rhino, Cook Medical, Bloomington, Indiana). ${ }^{9,10}$ During the procedure, subjects received sedation, analgesia, and a neuromuscular blockade if necessary. Percutaneous puncture of the trachea was done under clear fiberoptic visualization. The guide wire was inserted and advanced forward until approximately $1 \mathrm{~cm}$ below the carina. One dilator was in- troduced under fiberoptic control. The tracheal cannula was then inserted using another dilator as guide. The cuff of the tracheal cannula was inflated, and the guide wire was removed.

\section{Follow-Up}

Follow-up data of all 71 subjects are summarized in Table 3. Immediately after ICU discharge, 60 subjects $(84.5 \%)$ were referred to rehabilitation facilities. Four subjects went home with support of domestic home care, and 5 subjects were referred to a nursing facility. Following subjects' or families' wishes, 2 subjects were referred to another acute care center. In $16.9 \%$ of cases, subjects were referred with ongoing ventilator support. At the end of the study period, $36.6 \%$ of subjects had died, most of them (80.8\%) during the first 6 months after discharge. Table 4 shows follow-up results on the tracheostomy and final residency of survivors only $(n=45)$. During the 2-y course, $64.8 \%$ of all subjects had been decannulated. Most of the survivors $(88.9 \%)$ were successfully decannulated. Nearly half of all decannulations were done during the first month after discharge $(47.8 \%)$. Only 2 subjects remained with their initial tracheostomy. In 5 subjects $(7 \%)$, tracheostomy was modified to a surgical tracheostomy during the $2 \mathrm{y}$, but all except for one of these subjects died within the study period. Reasons were excessive granulations and tracheomalacia in 2 subjects, and in 3 subjects, long-term tracheostomies were felt to be necessary by an otolaryngologist. No medical rationales were given. No life-threatening adverse events, such as hypoxia or obstruction of the cannula, were reported in any of the 71 subjects. The neurological outcome measured by the Glasgow outcome scale was 3 points (interquartile range $1-4 ; n=68$ ). The median Glasgow outcome score of all survivors was 4 points, equaling "moderate disability" (interquartile range $3-4)$. Approximately $35 \%$ of the surviving subjects had only minor or moderate neurological and psychological deficits. Two subjects $(2.8 \%)$ were in a persistent vegetative state 2 y after the event.

When asked for complaints associated with the tracheostomy, $40.8 \%$ of subjects denied having any problems. Ten subjects (14.0\%) did not respond to the questionnaire. One subject was in a vegetative state, and information from caregivers was incomplete. Complaints stated by the remaining 31 subjects included dysphagia $(n=10)$, limited verbal communication $(n=7)$, dyspnea at rest and during exercise $(n=2$ each), hoarseness $(n=4)$, coughing $(n=5)$, excessive secretions $(n=4)$, unaesthetic scars $(n=4)$, foreign body sensation $(n=2)$, and stoma infections $(n=2)$. Subjects could choose multiple complaints. One subject presented with paralysis of the left vocal cord and unilateral dislocation of the arytenoid cartilage. Of the 2 subjects who still remained with their initial PDT until 
Table 2. Baseline Characteristics

\begin{tabular}{|c|c|c|c|}
\hline Characteristics & All Subjects $(N=71)$ & Survivors $(n=45)$ & Non-Survivors $(n=26)$ \\
\hline Age, mean \pm SD y & $61 \pm 19$ & $53.2 \pm 19.2$ & $74.8 \pm 7.8^{*}$ \\
\hline Male sex, $n(\%)$ & $44(62)$ & $15(57.7)$ & $29(64.4)$ \\
\hline $\mathrm{BMI}$, mean $\pm \mathrm{SD} \mathrm{kg}^{2} / \mathrm{cm}$ & $26 \pm 4$ & $25.7 \pm 4.1$ & $26.3 \pm 4.1$ \\
\hline $\mathrm{ICU}$ LOS, mean $\pm \mathrm{SD} d$ & $30 \pm 16$ & $27.2 \pm 11.3$ & $34.6 \pm 20.6$ \\
\hline Hospital LOS, mean $\pm \mathrm{SD} d$ & $35 \pm 21$ & $32.5 \pm 19.9$ & $39.9 \pm 22.0$ \\
\hline APACHE II score, mean \pm SD & $15.5 \pm 6.2$ & $13.6 \pm 5.8$ & $18.8 \pm 5.6^{*}$ \\
\hline SOFA score, mean $\pm \mathrm{SD}$ & $6.0 \pm 2.8$ & $6.2 \pm 2.8$ & $5.5 \pm 2.8$ \\
\hline SAPS II score, mean \pm SD & $35.1 \pm 12.9$ & $30.6 \pm 11.7$ & $43.2 \pm 10.9^{*}$ \\
\hline \multicolumn{4}{|l|}{ Primary reason for ICU admittance, $n(\%)$} \\
\hline CNS & $43(59.7)$ & $24(53.3)$ & $19(73)$ \\
\hline Multiple trauma & $12(16.9)$ & $10(22.2)$ & $2(7.7)$ \\
\hline Pulmonary (ARDS) & $6(8.5)$ & $4(8.9)$ & $2(7.7)$ \\
\hline Acute abdominal problems & $4(5.6)$ & $3(6.6)$ & $1(3.8)$ \\
\hline Post-surgery & $4(5.6)$ & $2(4.4)$ & $2(7.8)$ \\
\hline Cardiopulmonary resuscitation & $2(2.8)$ & $2(4.4)$ & $0(0)$ \\
\hline \multicolumn{4}{|l|}{ Tracheostomy } \\
\hline Ventilator days before PDT, mean $\pm \mathrm{SD} d$ & $8.4 \pm 3.4$ & $8.6 \pm 3.2$ & $8.1 \pm 3.9$ \\
\hline \multicolumn{4}{|l|}{ Reasons for PDT, $n(\%)$} \\
\hline Prolonged ventilatory support & $30(42.3)$ & $22(48.9)$ & $8(30.8)$ \\
\hline Weaning failure & $22(31.0)$ & $11(24.4)$ & $11(42.3)$ \\
\hline Ongoing neurological deficit & $19(26.7)$ & $12(26.7)$ & $7(26.9)$ \\
\hline $\begin{array}{l}\text { * Significant difference between survivors and non-survivors } \\
\text { BMI = body mass index } \\
\text { LOS = length of stay } \\
\text { APACHE II = Acute Physiology and Chronic Health Evaluat } \\
\text { SOFA = Sequential Organ Failure Assessment } \\
\text { SAPS = Simplified Acute Physiology Score } \\
\text { CNS = central nervous system } \\
\text { PDT = percutaneous dilatational tracheostomy }\end{array}$ & & & \\
\hline
\end{tabular}

the end of the follow-up, one was in a vegetative state. A tracheal stenosis was suspected, but otolaryngologists did not see the need for surgical intervention. The other subjects and/or caregivers did not report any complaints. The caregivers of the one subject who survived the follow-up and had a secondary surgical tracheostomy complained of recurrent infections of the stoma after surgical conversion. Before conversion, changing of the cannula was reported to be difficult, without an emergency situation being reported.

\section{Discussion}

In critical care medicine, bedside techniques for PDT are among the most common surgical procedures. Since its introduction to clinical medicine by Ciaglia et al, ${ }^{9}$ PDT has become more popular and has nearly replaced traditional surgical tracheostomy, especially in patients undergoing prolonged mechanical ventilation and suffering from weaning failure. ${ }^{7,9}$ Studies suggest that PDT is easier and quicker to perform and is associated with lower costs and fewer complications than the traditional procedure. ${ }^{11-13}$ In particular, surgical complications, such as major bleeding and local infections, can be reduced by PDT techniques, and cosmetic results seem to be superior. ${ }^{6,14}$ However, data on long-term outcomes of patients who have received a PDT are very limited, and surgical techniques are still the favored choice for a permanent tracheostomy.

In this study, we performed a 2-y follow-up in 71 subjects who had received PDT during their ICU stay and were discharged with the tracheal cannula in place. Subjects included in our study came from a mixed cohort of our interdisciplinary surgical ICU. The majority were admitted due to cerebral insults or multiple trauma. Reviewing the literature, this seems to be the most common cause for receiving an elective tracheostomy in a surgical cohort. ${ }^{13}$ Reasons for tracheostomy were the need for prolonged ventilator support, weaning failure, or ongoing neurological deficits, such as dysphagia and an assumed risk of aspiration. This is also in accordance with other centers' experiences. ${ }^{15}$

We performed tracheostomy via a single-dilator technique using the modified Seldinger technique described by Ciaglia et al. ${ }^{9}$ The modification consists of the application of only one curved dilator with a hydrophilic coating. ${ }^{10}$ 
Table 3. Follow-Up Data of All Subjects $(N=71)$

\begin{tabular}{|c|c|}
\hline Characteristics & $n(\%)$ \\
\hline 2-y mortality & $26(36.6)$ \\
\hline \multicolumn{2}{|l|}{ Referral to: } \\
\hline Rehabilitation facility & $60(84.5)$ \\
\hline Home & $4(5.6)$ \\
\hline Nursing facility & $5(7.0)$ \\
\hline Secondary hospital & $2(2.8)$ \\
\hline Referral on ventilatory support & $12(16.9)$ \\
\hline \multicolumn{2}{|l|}{ Decannulation } \\
\hline Total $(\%$ of $n=71)$ & $46(64.8)$ \\
\hline Within 2 weeks ( $\%$ of decannulations, $n=46$ ) & $12(26.1)$ \\
\hline Within 1 month ( $\%$ of decannulations, $n=46$ ) & $22(47.8)$ \\
\hline Within 6 months ( $\%$ of decannulations, $n=46$ ) & $36(78.3)$ \\
\hline Unknown state of tracheostomy & $8(11.3)$ \\
\hline Conversion in ST & $5(7.0)$ \\
\hline \multicolumn{2}{|l|}{ GOS } \\
\hline GOS 1 (death) & $26(36.6)$ \\
\hline GOS 2 & $2(2.8)$ \\
\hline GOS 3 & $15(21.1)$ \\
\hline GOS 4 & $13(18.3)$ \\
\hline GOS 5 & $12(16.9)$ \\
\hline Data missing & $3(4.2)$ \\
\hline \multicolumn{2}{|l|}{ Complaints } \\
\hline No complaints & $29(40.8)$ \\
\hline Dysphagia & $10(16.4)$ \\
\hline Foreign body sensation & $2(3.3)$ \\
\hline Disturbance of speech & $7(11.5)$ \\
\hline Hoarseness & $4(6.5)$ \\
\hline Scars & $4(6.5)$ \\
\hline Dyspnea on exercise & $2(3.3)$ \\
\hline Dyspnea during suction/cannula change & $2(3.3)$ \\
\hline Dyspnea in rest & $2(3.3)$ \\
\hline Excessive secretion & $4(6.5)$ \\
\hline Coughing & $5(8.2)$ \\
\hline Pneumonia & $2(3.3)$ \\
\hline Stoma infection & $2(3.3)$ \\
\hline Stridor & $3(4.9)$ \\
\hline Anosmia & $1(1.6)$ \\
\hline \multicolumn{2}{|l|}{ Feeding } \\
\hline Independently & $30(42.3)$ \\
\hline With help & $9(12.7)$ \\
\hline Feeding tube & $25(35.2)$ \\
\hline Unknown & $7(9.9)$ \\
\hline
\end{tabular}

Multiple-dilator tracheostomy and single-step dilation tracheostomy are associated with the lowest intra-procedural risks, such as technical difficulties and major bleeding events. ${ }^{6}$ The use of fiberoptic tracheoscopy as intratracheal visual guidance has reduced peri-procedural complication rates even further. ${ }^{16}$ Accordingly, single-step dilation tracheostomy has evolved as the most popular procedure used for tracheostomies today. ${ }^{7}$ The follow-up
Table 4. Follow-up Data From Survivors Only

\begin{tabular}{lc}
\hline \hline \multicolumn{1}{c}{ Characteristics } & Data \\
\hline Decannulated within 2-y follow-up, $n(\%)$ & $40(88.9)$ \\
Open tracheostomy, $n(\%)$ & $3(6.7)$ \\
$\quad$ Conversion to ST & $1(2.2)$ \\
PDT as initially done & $2(4.4)$ \\
Unknown & $2(4.4)$ \\
Final residency, $n$ & \\
$\quad$ Home & 28 \\
$\quad$ Nursing facility & 16 \\
$\quad$ Unknown & 1 \\
& \\
$n=45$. & \\
ST $=$ surgical tracheostomy & \\
PDT $=$ percutaneous dilatational tracheostomy & \\
\hline
\end{tabular}

data of our study might therefore be comparable with many other patient collectives.

In our cohort of subjects with PDT, we found an inhospital mortality of $32 \%$. Mortality rates in this mixed surgical cohort are not very easy to compare, but traumatic brain injury alone is associated with mortality rates between 29 and 55\%, whereas trauma patients usually show slightly lower mortality rates. ${ }^{17,18}$ During the study period of $2 \mathrm{y}$, another $36 \%$ of our subjects died, resulting in a $2-\mathrm{y}$ mortality of $63.4 \%$. Antonelli et al ${ }^{19}$ followed subjects for $1 \mathrm{y}$ after surgical or dilatational tracheostomy. The authors found a mortality rate $1 \mathrm{y}$ after discharge as high as $45 \%$, finally reaching a $1-y$ mortality of $63 \%$. Long-term survival analyses of subjects who had received a tracheostomy also have reported mortality rates between 52 and $67 \% .{ }^{20,21}$

After discharge, we found that a large number of the subjects in our study were decannulated in their course of recovery. More than half were decannulated during the first month after PDT, and $88.9 \%$ of all survivors remained decannulated. Only 2 subjects remained with their cannula as discharged from the ICU. Five subjects received a conversion to a surgical tracheostomy. However, only 2 of these had complaints related to their tracheostomy beforehand. In the 3 remaining subjects, health-care providers felt the conversion to be necessary as a prophylactic procedure. Reasons given were actually based on a traditional view of tracheostomies. In our study, subjects remaining with their initial PDT did not have severe complications. Acute complications associated with the PDT procedure are well described and include bleeding, malpositioning, and pneumothorax. ${ }^{6}$ However, late complications are much less frequently reported. ${ }^{19,22,23}$ Tracheal lesions that may result in tracheal stenosis or tracheomalacia are known as long-term risks of percutaneous tracheostomy. ${ }^{6}$ Both may lead to life-threatening complications during cannula changes or may affect quality of life later on. However, 
only a very few studies have addressed these issues, and it seems that tracheal stenosis is even more frequent in patients who have received a surgical tracheostomy. ${ }^{24}$ In a study by Kiran et $\mathrm{al}^{25}$ on PDT subjects, only $28.8 \%$ were decannulated, and $13.3 \%$ received a so-called "permanent tracheostomy" (surgical conversion) within the 6-month follow-up. The authors give no information concerning reason for conversion and outcome of decannulated or converted cases. Antonelli et al ${ }^{19}$ report of an equivalent low rate of conversion to surgical tracheostomy. The authors report of an equivalent low rate of conversion. Four subjects requiring stomatoplasty; three due to tracheal stenosis and granuloma and one without such complications. ${ }^{19}$ Melloni et al ${ }^{22}$ followed 15 subjects after PDT for 6 months after discharge. Two showed tracheal lesions, but no surgical intervention was required. One could be decannulated, and one remained with the cannula due to a persistent vegetative state. No data are given regarding the frequency of decannulation or the comfort or quality of life in this study.

Personal complaints connected to the tracheostomy seemed to be quite frequent in our study, with nearly $60 \%$ reporting at least one symptom. There was a large group of complaints that affected quality of life substantially like dysphagia, dysphonia, or dyspnea.

However, none of these symptoms can be definitively attributed to the method of tracheostomy because a comparison of different methods has not been done in this study. Dysphagia and dysphonia as well as reduced Glasgow outcome score might be attributable to the initial central nervous system damage. Additionally, long-term mechanical ventilation and critical illness are independent risk factors for the development of neurological dysfunctions like dysphagia. ${ }^{26,27}$ Dyspnea might have multiple causes. Most subjects reported short incidences of dyspnea when suction was applied via the tracheostomy or during cannula changes. In one subject, dyspnea was clearly associated with the detected granulation of the trachea, and in another, it was associated with the paralysis of one vocal cord. Dyspnea unrelated to suctioning events appeared in only $6.6 \%$ of cases. In a large study on PDT and surgical tracheostomy by Silvester et al, ${ }^{28}$ dyspnoe was described by $28 \%$ and cough by as many as $65 \%$ of all patients after PDT.

Dysphagia, dyspnea, dysphonia, and unaesthetic scars may all be found in PDT as well as in surgical tracheostomy. ${ }^{28}$ However, significant differences are described for the length of the scars only. ${ }^{28}$ In our study, we found $6.6 \%$ of subjects mentioning the scar as a relevant problem. A comparison with other studies is difficult because we did not inspect or judge the size or appearance of the scars ourselves.

There are multiple limitations to our study. Although this represents the largest long-term follow-up to date in the literature, the number of included subjects is rather small and from a single center only. Future studies should include randomization between surgical tracheostomy and PDT as well as a tracheal fiberoptic examination. Therefore, the results should be interpreted with caution.

\section{Conclusions}

In our study, we were able to follow 71 subjects who had received a PDT for 2 years after hospital discharge and evaluate the long-term outcome of PDT. To our knowledge, this is the largest group who has undergone a 2-y follow-up. We found that most subjects could successfully be decannulated within the first 6 months. There was a high frequency of common symptoms after tracheostomy and very few subsequent surgical conversions of tracheostomy.

Discharge of subjects after PDT seems to be safe as we found no severe post-discharge complications in our cohort. Therefore, PDT might be a possible alternative to the surgical approach even for patients with expected longterm cannulation. However, this hypothesis should be tested in a prospective study.

\section{REFERENCES}

1. Karanjia N, Nordquist D, Stevens R, Nyquist P. A clinical description of extubation failure in patients with primary brain injury. Neurocrit Care 2011;15(1):4-12.

2. Ko R, Ramos L, Chalela JA. Conventional weaning parameters do not predict extubation failure in neurocritical care patients. Neurocrit Care 2009;10(3):269-273.

3. Lazaridis C, DeSantis SM, McLawhorn M, Krishna V. Liberation of neurosurgical patients from mechanical ventilation and tracheostomy in neurocritical care. J Crit Care 2012;27(4):417.e1-417.e8.

4. Andriolo BN, Andriolo RB, Saconato H, Atallah ÁN, Valente O. Early versus late tracheostomy for critically ill patients. Cochrane Database Syst Rev 2015;(1):CD007271.

5. Alali AS, Scales DC, Fowler RA, Mainprize TG, Ray JG, Kiss A, et al. Tracheostomy timing in traumatic brain injury: a propensitymatched cohort study. J Trauma Acute Care Surg 2014;76(1):70-76; discussion 76-78.

6. Putensen C, Theuerkauf N, Guenther U, Vargas M, Pelosi P. Percutaneous and surgical tracheostomy in critically ill adult patients: a meta-analysis. Crit Care 2014;18(6):544.

7. Vargas M, Sutherasan Y, Antonelli M, Brunetti I, Corcione A, Laffey JG, et al. Tracheostomy procedures in the intensive care unit: an international survey. Crit Care 2015;19:291.

8. Jennett B, Bond M. Assessment of outcome after severe brain damage. Lancet Lond Engl 1975;1(7905):480-484.

9. Ciaglia P, Firsching R, Syniec C. Elective percutaneous dilatational tracheostomy: a new simple bedside procedure; preliminary report. Chest 1985;87(6):715-719.

10. Byhahn C, Wilke HJ, Halbig S, Lischke V, Westphal K. Percutaneous tracheostomy: ciaglia blue rhino versus the basic ciaglia technique of percutaneous dilational tracheostomy. Anesth Analg 2000; 91(4):882-886.

11. Bacchetta MD, Girardi LN, Southard EJ, Mack CA, Ko W, Tortolani $\mathrm{AJ}$, et al. Comparison of open versus bedside percutaneous dilata- 


\section{Percutaneous Dilatational Tracheostomy}

tional tracheostomy in the cardiothoracic surgical patient: outcomes and financial analysis. Ann Thorac Surg 2005;79(6):1879-1885.

12. Grover A, Robbins J, Bendick P, Gibson M, Villalba M. Open versus percutaneous dilatational tracheostomy: efficacy and cost analysis. Am Surg 2001;67(4):297-301; discussion 301-302.

13. Oggiano M, Ewig S, Hecker E. A comparison of percutaneous dilatational tracheostomy versus conventional surgical tracheostomy. Pneumologie 2014;68(5):322-328.

14. Brass P, Hellmich M, Ladra A, Ladra J, Wrzosek A. Percutaneous techniques versus surgical techniques for tracheostomy. Cochrane Database Syst Rev 2016;(7):CD008045.

15. Cheung NH, Napolitano LM. Tracheostomy: epidemiology, indications, timing, technique, and outcomes. Respir Care 2014;59(6):895915; discussion 916-919.

16. Peris A, Linden M, Pellegrini G, Anichini V, Di Filippo A. Percutaneous dilatational tracheostomy: a self-drive control technique with video fiberoptic bronchoscopy reduces perioperative complications. Minerva Anestesiol 2009;75(1):21-25.

17. Chico-Fernández M, Llompart-Pou JA, Guerrero-López F, SánchezCasado M, García-Sáez I, Mayor-García MD, et al. Epidemiology of severe trauma in Spain: registry of trauma in the ICU (RETRAUCI): pilot phase. Med Intensiva 2016;40(6):327-347.

18. Peeters W, van den Brande R, Polinder S, Brazinova A, Steyerberg EW, Lingsma HF, Maas AI. Epidemiology of traumatic brain injury in Europe. Acta Neurochir 2015;157(10):1683-1696.

19. Antonelli M, Michetti V, Di Palma A, Conti G, Pennisi MA, Arcangeli A, et al. Percutaneous translaryngeal versus surgical tracheostomy: a randomized trial with 1-yr double-blind follow-up. Crit Care Med 2005;33(5):1015-1020.
20. Scales DC, Thiruchelvam D, Kiss A, Redelmeier DA. The effect of tracheostomy timing during critical illness on long-term survival. Crit Care Med 2008;36(9):2547-2557.

21. Young D, Harrison DA, Cuthbertson BH, Rowan K, TracMan Collaborators. Effect of early vs late tracheostomy placement on survival in patients receiving mechanical ventilation: the TracMan randomized trial. JAMA 2013;309(20):2121-2129.

22. Melloni G, Muttini S, Gallioli G, Carretta A, Cozzi S, Gemma M, Zannini P. Surgical tracheostomy versus percutaneous dilatational tracheostomy: a prospective-randomized study with long-term follow-up. J Cardiovasc Surg 2002;43(1):113-121.

23. Simon M, Metschke M, Braune SA, Püschel K, Kluge S. Death after percutaneous dilatational tracheostomy: a systematic review and analysis of risk factors. Crit Care 2013;17(5):R258

24. Dempsey GA, Morton B, Hammell C, Williams LT, Tudur Smith C, Jones T. Long-term outcome following tracheostomy in critical care: a systematic review. Crit Care Med 2016;44(3):617-628.

25. Kiran S, Eapen S, Chopra V. A comparative study of complications and long term outcomes of surgical tracheostomy and two techniques of percutaneous tracheostomy. Indian J Crit Care Med 2015;19(2): 82-86.

26. Macht M, White SD, Moss M. Swallowing dysfunction after critical illness. Chest 2014;146(6):1681-1689.

27. Tolep K, Getch CL, Criner GJ. Swallowing dysfunction in patients receiving prolonged mechanical ventilation. Chest 1996;109(1):167172.

28. Silvester W, Goldsmith D, Uchino S, Bellomo R, Knight S, Seevanayagam S, et al. Percutaneous versus surgical tracheostomy: A randomized controlled study with long-term follow-up. Crit Care Med 2006;34(8):2145-2152. 